\title{
Comparative Study between Modified William's Airway (Fekry Airway) versus the U Shaped Guedl's Airway as Assisted Device for Fiber Optic Intubation: A Randomized Controlled Trial
}

Ahmed Abdalla Mohamed ( $\square$ ahmed.aboali7268@gmail.com )

Cairo University https://orcid.org/0000-0002-3024-7974

Sherin Refaat Mahmoud

Cairo University

Maha Mohamed Ismail Youssef

Cairo University

Nasser Mohamed Dobal

Cairo University

Heba Alla Hussein Zoheir

Cairo University

Hany Mohamed El Hady

Cairo University

Ahmed Mohamed Elbadawy

Cairo University

Tamer Mohamed Khair

Cairo University

Mohamed Ibrahim Belita

Cairo University

Ehab Mohi Atta

Cairo University

Ahmed Abd EL Aziz Seleem

Cairo University

Safinaz Hassan Osman

Cairo University

Rania Samir Fahmy

Cairo University

Reham Ali Abdelrahman

Cairo University

Ahmed Essam Salem

Tanta University 


\section{Research Article}

Keywords: Modified William's airway, U shaped Guedl's airway, Fiber Optic Intubation

Posted Date: December 21st, 2018

DOI: https://doi.org/10.21203/rs.2.139/v1

License: (c) (1) This work is licensed under a Creative Commons Attribution 4.0 International License. Read Full License 


\section{Abstract}

The authors have removed this preprint from Research Square. 